\title{
Long non-coding RNA SNHG1 contributes to cisplatin resistance in non-small cell lung cancer by regulating miR-140-5p/Wnt/ $\beta$-catenin pathway
}

\author{
S. L. SHI ${ }^{1}$, Z. H. ZHANG ${ }^{2, *}$ \\ ${ }^{1}$ Department of Respiratory Medicine, Xian No.1 Hospital, Xian, Shaanxi, China; ${ }^{2}$ Department of Respiratory Medicine, Xian XD Group Hos- \\ pital, No. 97, Xian, Shaanxi, China \\ *Correspondence: caicai16688@163.com
}

Received December 18, 2018 / Accepted April 3, 2019

\begin{abstract}
Small nucleolar RNA host gene 1 (SNHG1) has been identified to function as an oncogene in a large number of human cancers. Nevertheless, the biologic role and underlying molecular mechanism of SNHG1 on cisplatin (DDP)-resistance in NSCLC is still unknown. qRT-PCR assay was performed to assess the expression levels of SNHG1 and miR-140-5p. Western blot analysis was used to determine Wnt1, cyclinD1, c-Myc and $\beta$-catenin levels. The direct correlation between SNHG1 and miR-140-5p was verified by dual-luciferase reporter assay and RNA immunoprecipitation (RIP) assay. CCK-8 assay and Transwell assay were applied to determine cell proliferation ability, and cell migration and invasion capacities, respectively. Tumor xenograft was performed to confirm the effect of SNHG1 on DDP-resistance of NSCLC in vivo. Our data showed SNHG1 was upregulated in DDP-resistant NSCLC tissues and cell lines. SNHG1 knockdown suppressed the proliferation, migration, invasion and DDP-resistance in DDP-resistant NSCLC cell lines in vitro and inhibited tumor growth in vivo. Moreover, SNHG1 repressed miR-140-5p expression by directly binding to miR-140-5p. SNHG1-knockdown-mediated regulatory effect was antagonized by miR-140-5p. Furthermore, Wnt/ $\beta$-catenin signaling was involved in SNHG1/miR-140$5 \mathrm{p}$-mediated regulation in DDP-resistance of NSCLC cell lines. The results suggested that SNHG1 knockdown ameliorated DDP-resistance of NSCLC by regulating miR-140-5p/Wnt/ $\beta$-catenin pathway, providing a new potential therapeutic target for DDP-resistance NSCLC treatment.
\end{abstract}

Key words: small nucleolar RNA host gene 1 (SNHG1), miR-140-5p, Wnt/ß-catenin pathway, DDP-resistance

Lung cancer is the leading cause of cancer-related death around the world [1]. Non-small cell lung cancer (NSCLC), which includes squamous cell carcinoma, adenocarcinoma and large-cell carcinoma, accounts for approximately $80-85 \%$ of all lung cancer cases [2]. Cisplatin (DDP) is one of the most effective DNA-damaging anticancer drugs, which has been widely used for the treatment of various human malignancies, including NSCLC [3]. However, the development of DDP-resistance has been reported to undermine its favorable therapeutic effects and lead to poor prognosis in NSCLC patients [4]. Therefore, it is of importance to explore the molecular mechanism of DDP-resistance and provide effective targeting therapeutic strategies for DDP-resistance NSCLC treatment.

Long non-coding RNAs (lncRNAs), a novel heterogeneous class of transcripts $>200 \mathrm{nt}$ in length with no proteincoding potential, constitute an important layer of transcriptional regulation [5]. Growing amount of evidence has suggested that aberrant lncRNA expression is a major deter- minant of multiple human diseases, including cancers [6]. Recently, some lncRNAs have been demonstrated to influence the chemoresistance in NSCLC, highlighting their role as potential therapeutic targets for DDP-resistance NSCLC therapy $[7,8]$. Small nucleolar RNA host gene 1 (SNHG1), a nuclear-enriched lncRNA localized at chromosome 11q12.3, has been identified as an oncogene in a large number of human cancers [9]. For instance, upregulation of SNHG1 contributed to the progression of glioma and predicted poor prognosis [10]. SNHG1 promoted the aggravation of hepatocellular carcinoma through directly repressing miR-195 expression [11], and accelerated osteosarcoma progression by sponging miR-577 and regulating $\mathrm{Wnt} / \beta$-catenin signaling [12]. Additionally, SNHG1 was upregulated in NSCLC tissues and cells and its knockdown repressed the proliferation of NSCLC cells [13]. Moreover, upregulation of SNHG1 contributed to the progression of NSCLC by sponging miR-145-5p and regulating MTDH expression [14]. Nevertheless, the biologic role and underlying molec- 
ular mechanism of SNHG1 on DDP-resistance in NSCLC is still unknown.

MicroRNAs (miRNAs), a type of small evolutionarily conserved non-coding RNAs with 19-24 nt in length, function as regulators of gene expression and thus implicate in fundamental cellular processes, such as development, differentiation, survival and death [15]. Accumulating evidence shows that dysregulation of miRNAs is strongly linked to tumorigenesis, cancer progression and chemoresistance [16]. Recently, the competing endogenous RNAs (ceRNAs) hypothesis proposed that lncRNAs might directly interact with target miRNAs and regulate miRNA and/or gene expression [17]. Moreover, the ceRNAs concept has been described to play an important role in the chemoresistance of cancers [18]. In this study, we found that SNHG1 was upregulated in DDP-resistant NSCLC tissues and cell lines. Furthermore, SNHG1 knockdown ameliorated DDP-resistance of NSCLC by acting as a ceRNA of miR-140-5p and regulating $\mathrm{Wnt} / \beta$-catenin pathway, providing a new potential therapeutic target for DDP-resistance NSCLC treatment.

\section{Materials and methods}

Clinical samples. Thirty-one cases of DDP-sensitive NSCLC tissues and DDP-resistant NSCLC tissues were obtained from 62 NSCLC patients who underwent standard DDP-based chemotherapy at Xi'an No.1 Hospital with prior informed consents. All the tissues were stored at $-80^{\circ} \mathrm{C}$ until RNA extraction. Patients with no significant clinical efficacy or progression disease were defined as DDP-resistance after 2 cycles of DDP-based chemotherapy according to the computed tomography (CT) scanning and Response Evaluation Criteria in Solid Tumors (RECIST) criteria. The study was approved by the Ethics Committee of Xian No.1 Hospital.

Cell culture and treatment. Human bronchial epithelial cell line (16HBE) and NSCLC cell lines (A549 and H1299) purchased from ATCC (Manassas, VA, USA) were grown in RPMI-1640 medium (Hycolone laboratories, Logan, UT, USA) supplemented with $10 \%$ fetal bovine serum (FBS, Gibco, Rockville, MD, USA), 0.5\% penicillin-streptomycin (Gibco) in a fully humidified atmosphere at $37^{\circ} \mathrm{C}$ containing $5 \% \mathrm{CO}_{2}$.

To construct DDP-resistant NSCLC cell lines (A549/DDP and H1299/DDP), A549 and H1299 cells were stimulated with a stepwise increasing concentration of DDP (Haosen, Jiangsu, China) over 6 months, as described previously [19]. For maintaining the drug-resistant phenotype of A549/DDP and H1299/DDP, additional $2 \mu \mathrm{M}$ of DDP was added into the culture medium.

Cell transfection. Twenty-five nM of siRNA targeting SNHG1 (si-SNHG1, GenePhama, Shanghai, China) or negative control (si-NC, GenePhama), $50 \mathrm{nM}$ of miRNA mimics (miR-140-5p mimics, GenePhama) or its control (miR-NC mimics, GenePhama), or $50 \mathrm{nM}$ of miRNA inhibi- tors (anti-miR-140-5p or anti-miR-NC, GenePhama) was transfected into cells using Lipofectamine 3000 reagent (Life Technologies, Carlsbad, CA, USA) according to the manufacturers' protocols. SNHG1 overexpression plasmid (pcDNA-SNHG1) also was commercially constructed by GenePhama, and pcDNA was used as negative control. $10 \mathrm{ng}$ of pcDNA-SNHG1 or pcDNA was transfected into cells by Lipofectamine 3000 reagent, and the stable transfected cells were selected with $1 \mu \mathrm{g} / \mathrm{ml}$ puromycin (Gibco).

Quantitative real-time PCR (qRT-PCR). RNA was extracted from tissues and cells with a mirVana miRNA Isolation Kit (Ambion, Austin, TX, USA) according to the manufacturers' protocols. The concentration of RNA extracts was measured by the NanoDrop spectrophotometer (Thermo Fisher Scientific, Waltham, MA, USA). Then, $1 \mu \mathrm{g}$ of RNA was reversely transcribed into cDNA using the M-MLV Reverse Transcriptase Kit (Invitrogen, Carlsbad, CA, USA) and qRT-PCR was performed using SYBR Green PCR Master Mix (Applied Biosystems, Foster city, CA, USA) on a Bio-Red IQ5 Multi-color RT-PCR Detection System (Bio-Red, Hercules, CA, USA). The relative expression of SNHG1 was evaluated by $2^{-\Delta \Delta \mathrm{Ct}}$ method and normalized to GAPDH expression. MiR-140-5p expression was performed by using TaqMan miRNA Reverse Transcription Kit (Applied Biosystems) and Taqman MicroRNA Assay Kit (Thermo Fisher Scientific) with U6 as an endogenous control.

Dual-luciferase reporter assay. SNHG1 wild-type or mutant-type reporter plasmid (SNHG1-WT or SNHG1MUT) containing the predicted binding sites of miR-140-5p or mutated miR-140-5p binding sites was constructed by GenePhama and transfected into cells, together with miR-NC mimics or miR-140-5p mimics. After $48 \mathrm{~h}$ transfection, the relative luciferase activity was measured with a Dual-Luciferase Reporter Assay System (Promega, Fitchburg, WI, USA).

RNA immunoprecipitation (RIP) assay. RIP assay was used to verify the endogenous connection between SNHG1 and miR-140-5p in treated cells using a Magna RIP Immunoprecipitation Kit (Millipore, Billerica, MA, USA). Briefly, cells transfected with miR-140-5p mimics were lysed using RIP lysis buffer (Takara, Beijing, China), and the lysates were incubated with RIP buffer containing magnetic beads conjugated with anti-Agol (Abnova, Taiwan, China) or isotypic anti-IgG (Abnova). Following digesting protein with Proteinase K (Merck, Darmstadt, Germany), qRT-PCR assay was performed to assess the enrichment of SNHG1 in immunoprecipitated RNA.

Colony formation assay. Cells were cultured in 6-well plate for $24 \mathrm{~h}$, and then transfected with si-NC, si-SNHG1, si-SNHG1+anti-miR-NC and si-SNHG1+anti-miR-140-5p. After 10 days, colonies were fixed with $4 \%$ paraformaldehyde (Applied Biosystems) and stained with $0.1 \%$ crystal violet (Applied Biosystems). The number of colonies was counted under a microscope (Leica, Wetzlar, Germany). 
Cell proliferation assay. Cell proliferation ability was assessed using Cell Counting Kit-8 (CCK-8, Dojindo, Tokyo, Japan) according to the instructions of manufacturers. In brief, at $0,24,48$ and $72 \mathrm{~h}$ after transfection, $10 \mu \mathrm{l}$ of CCK- 8 solution was added into each well and incubated at $37^{\circ} \mathrm{C}$ for $2 \mathrm{~h}$. The absorbance was measured at $450 \mathrm{~nm}$ by a microplate reader (Bio-Rad).

For IC50 determination, cells were exposed to different concentration of DDP $(1,10,20,40,80,160 \mu \mathrm{M})$ for $48 \mathrm{~h}$. Afterward, cells were incubated with CCK-8 solution and the absorbance was measured.

Transwell assay. Cell migration ability was measured with a Transwell-chamber culture system (Becton Dickinson, Franklin Lakes, NJ, USA), and cell invasion capacity was assessed using Matrigel-coated Transwell chamber (Becton Dickinson). After $48 \mathrm{~h}$ post-transfection, $200 \mu \mathrm{l}$ of serum-free medium containing $1.0 \times 10^{5}$ transfected cells were seeded into the upper chamber, and $500 \mu \mathrm{l}$ of growth medium containing $10 \%$ FBS was added into the lower chamber. Following $24 \mathrm{~h}$ of incubation, the cells that migrated or invaded to the lower surface of membrane were fixed with $4 \%$ paraformaldehyde and stained with $0.1 \%$ crystal violet. The images were taken and the numbers of migrated or invaded cells were counted using a microscope with an ImageJ software (National Institutes of Health, Bethesda, MD, USA).

Western blot. Cells were lysed by RIP lysis buffer and protein samples were obtained by centrifugation. An equal amount of each protein sample was separated on a $10 \%$ SDS-PAGE gel and transferred onto a PVDF membrane (Millipore). Blocked by $5 \%$ nonfat milk, the membranes were incubated with anti-Wnt1 (1:1000, Abcam, Cambridge, UK), anti-cyclinD1 (1:200, Abcam), anti-c-Myc (1:1000, Abcam), anti- $\beta$-catenin (1:5000, Abcam) and anti-GAPDH (1:1000, Abcam), followed by a further incubation with HRP-conjugated secondary antibodies (1:5000, Abcam). The protein bands were visualized using ECL Plus Western Blotting Detection System (National Institutes of Health).

sh-SNHG1-cells construct. Lentiviruses containing the sequences of SNHG1 knockout or negative control
(sh-SNHG1 or sh-NC) were commercially constructed by Applied Biosystems. A549/DDP cells were infected by sh-SNHG1 or sh-NC. One day after infection, the cells were treated with puromycin to derive the stably transfected cells.

In vivo assay. Female SCID mice (6-8 weeks) were purchased from Beijing Vital River Laboratory Animal Technology Corporation (Beijing, China) and housed under specific-pathogen-free condition. Approximately $5.0 \times 10^{6}$ A549/DDP cells stably transfected with lentivirus-mediated sh-SNHG1 or sh-NC were subcutaneously injected into nude mice $(n=10)$. At 3 days after implantation, an intravenous administration of DDP solution ( $3 \mathrm{mg} / \mathrm{kg}$ ) into each mouse was performed every 5 days. 5 weeks later, xenograft mice were euthanized for tumor excision. All the animal experiments were performed with the approval of Animal Research Committee of Xi'an No.1 Hospital.

Statistical analysis. All data were analyzed with SPSS 19.0 software (SPSS Inc., Chicago, IL, USA) and expressed as the mean \pm standard deviation (SD). A paired Student's t-test was used to compare the difference between two groups and the one-way ANOVA was performed to analyze multiple groups' differences. A value of $\mathrm{p}<0.05$ was considered statistically significant.

\section{Results}

Upregulation of SNHG1 in DDP-resistant NSCLC tissues and cell lines. To explore the effect of SNHG1 on DDP-resistance in NSCLC, qRT-PCR assay was initially performed to observe the expression of SNHG1 in DDP-resistant or -sensitive NSCLC tissues. As presented in Figure 1A, SNHG1 was significantly upregulated in DDP-resistant NSCLC tissues compared with DDP-sensitive NSCLC tissues. Then, we detected SNHG1 expression in NSCLC cell lines and human bronchial epithelial cell line. qRT-PCR assay revealed a significant upregulation of SNHG1 expression in NSCLC cells compared with that of control (Figure 1B). Moreover, compared with DDP-sensitive controls, SNHG1 was higher in DDP-resistant NSCLC cell lines (Figure 1B).
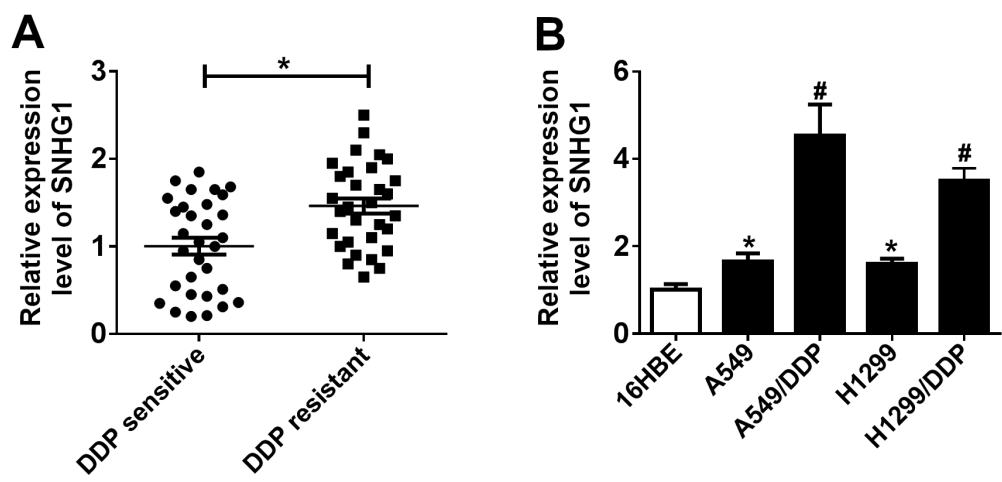

Figure 1. SNHG1 was upregulated in DDP-resistant NSCLC tissues and cell lines. qRT-PCR assay was used to assess the expression of SNHG1 in A) 31 cases of DDP-resistant NSCLC tissues and DDP-sensitive NSCLC tissues, B) human bronchial epithelial cells (16HBE), DDP-sensitive NSCLC cells (A549 and H1299) and DDP-resistant NSCLC cells (A549/DDP and H1299/DDP). * ${ }^{*}<0.05$ or ${ }^{*}$ p $<0.05$ vs. respective control. 
All these results supported that SNHG1 might play a vital role in DDP-resistant NSCLC.

si-SNHG1 suppressed the proliferation, migration, invasion and DDP-resistance in DDP-resistant NSCLC cell lines. Then, we measured the IC50 of DDP in DDP-resistant NSCLC cell lines compared to parental NSCLC cell lines. These data revealed that IC50 of DDP in DDP-resistant NSCLC cell lines was higher than that in parental cell lines (Figures $2 \mathrm{~A}$ and $2 \mathrm{~B}$ ). To further explore the function of SNHG1 on DDP-resistant NSCLC, we manipulated SNHG1 expression in A549/DDP and H1299/DDP cells by transfection with siRNA targeting SNHG1 (si-SNHG1). As shown in Figure 2C, SNHG1 expression was highly weakened by introduction si-SNHG1 in A549/DDP and H1299/DDP cells. Moreover, IC50 of DDP were highly reduced following SNHG1 knockdown in A549/DDP and H1299/DDP cells (Figure 2D). Further functional experiments demonstrated that SNHG1 knockdown led to a significant suppression of cell colony formation (Figure 2E), cell proliferation ability
(Figures $2 \mathrm{~F}$ and $2 \mathrm{G}$ ), migration ability (Figure $2 \mathrm{H}$ ) and invasion capacity (Figure 2I) in A549/DDP and H1299/DDP cells. Taken together, all these data indicated that SNHG1 might promote the DDP-resistance of NSCLC.

SNHG1 repressed miR-140-5p expression by directly binding to miR-140-5p. To further investigate the underlying regulatory mechanism of SNHG1 on DDP-resistance of NSCLC, bioinformatics prediction softwares were used to predict the directly interactional miRNAs of SNHG1. The predicted data demonstrated that SNHG1 might directly interact with miR-140-5p (Figure 3A). To confirm that, dualluciferase reporter assay and RIP assay were performed. For luciferase reporter assay, SNHG1 wild-type or mutant-type reporter plasmid (SNHG1-WT or SNHG1-MUT) was transfected into DDP-resistant NSCLC cells together with miR-NC mimics or miR-140-5p mimics. The results indicated that the luciferase activity of SNHG1-WT was dramatically reduced by transfection with miR-140-5p mimics in A549/DDP cells (Figure 3B) and H1299/DDP cells (Figure 3C). Whereas,
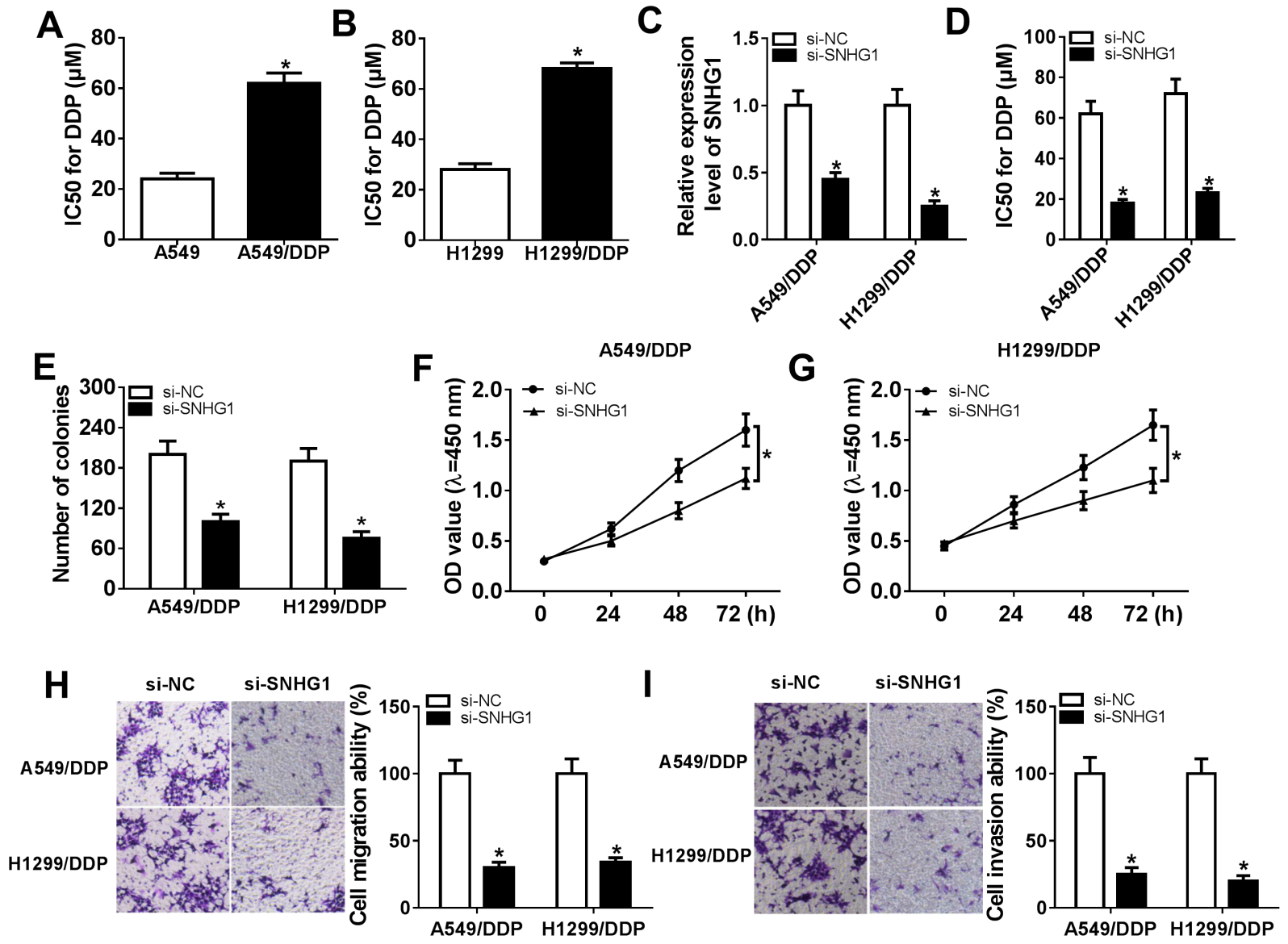

Figure 2. si-SNHG1 suppressed cell proliferation, migration, invasion and DDP-resistance in NSCLC cell lines. A549 and A549/DDP (A), H1299 and H1299/DDP (B) cells were exposed to different concentrations of DDP $(1,10,20,40,80,160 \mu \mathrm{M})$ for $48 \mathrm{~h}$, followed by the detection of the calculation of IC50 of cisplatin by CCK-8 assay. A549/DDP and H1299/DDP cells were transfected with si-NC or si-SNHG1, followed by the measurement of SNHG1 expression by qRT-PCR assay (C), IC50 of DDP by CCK-8 assay (D), cell colony formation by colony formation assay (E), cell proliferation ability by CCK-8 assay (F) and (G), cell migration (H) and invasion (I) capacities by Transwell assay. ${ }^{*} \mathbf{p}<0.05$ vs. A549/H1299 or si-NC. 
little change was found in the luciferase activity of SNHG1MUT in the presence of miR-140-5p mimics (Figures 3B and 3C). For RIP assay, anti-Agol or anti-IgG was used in the lysates of A549/DDP and H1299/DDP cells transfected with miR-140-5p mimics. The data revealed that SNHG1 was specifically recruited to the miRNP complex isolated with anti-Agol in response to the upregulation of miR-140-5p
(Figure 3D). Further, we observed whether SNHG1 could regulate miR-140-5p expression in A549/DDP and H1299/ DDP cells by transfecting with SNHG1 overexpression vector (pcDNA-SNHG1) or si-SNHG1. qRT-PCR assays showed that SNHG1 level was strikingly upregulated when transfected with pcDNA-SNHG1, while it was prominently downregulated by si-SNHG1 introduction (Figure 3E).

\section{A hsa-miR-140-5p SNHG1 WT SNHG1 MUT}

\section{3' GAUGguaUCCCAUUUUGGUAC 5' 5' ...AGACCUUGUAG-AUAACCACUC... 3' 5' ...AGUGGUUGUAG-AUUUGGUGAC... 3'}

B
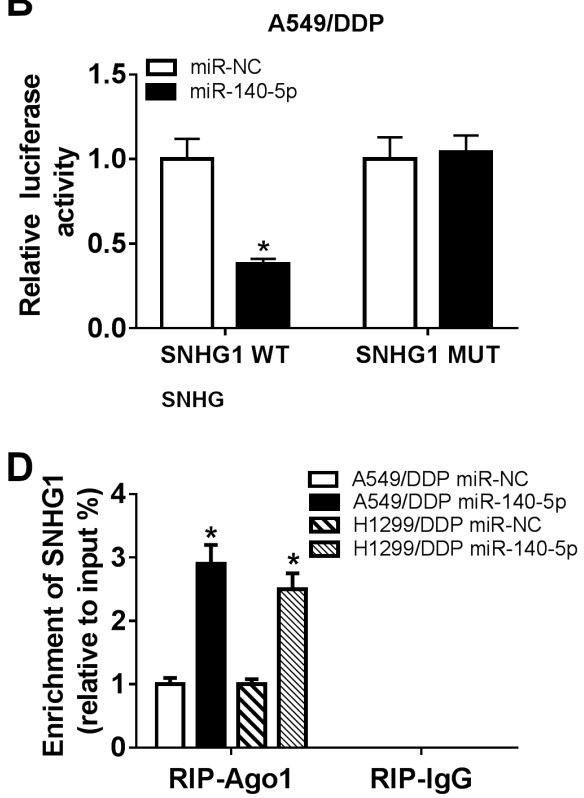

$\mathbf{F}$

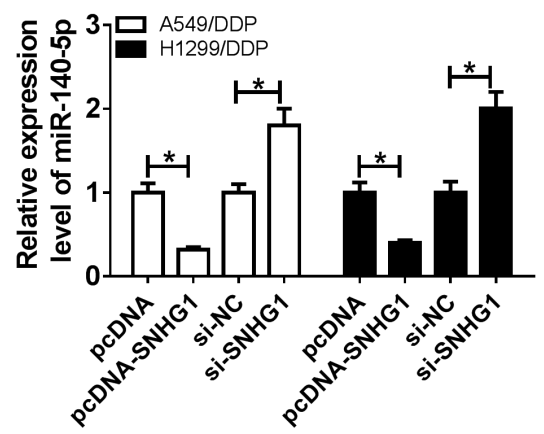

C

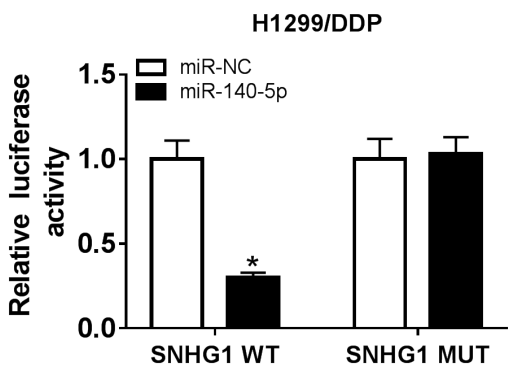

E

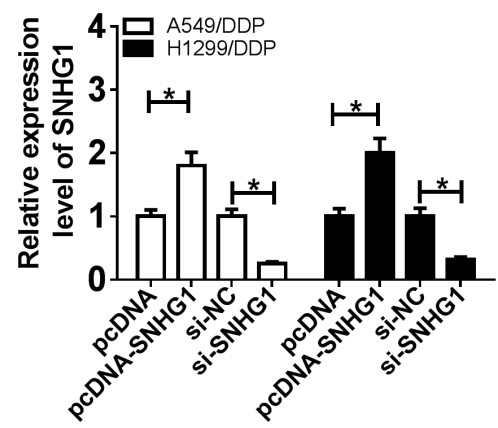

G

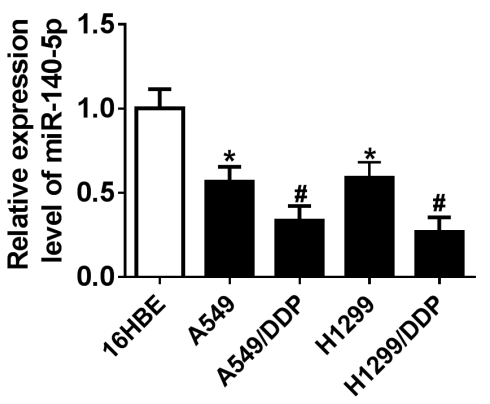

Figure 3. SNHG1 bound to miR-140-5p and repressed its expression. A) The predicted binding sites between SNHG1 and miR-140-5p and the mutation in predicted seed region. A549/DDP cells (B) and H1299/DDP cells (C) were co-transfected with SNHG1-WT or SNHG1-MUT and miR-NC mimics or miR-140-5p mimics, followed by the determination of relative luciferase activity. D) RNA immunoprecipitation (RIP) assay was performed to verify whether SNHG1 endogenous interacted with miR-140-5p in A549/DDP and H1299/DDP cells transfected with miR-140-5p. pcDNA, pcDNA-SNHG1, si-NC or si-SNHG1 was transfected into A549/DDP and H1299/DDP cells, followed by the detection of SNHG1 (E) and miR-140-5p (F) expression levels by qRT-PCR. (G) qRT-PCR assay of miR-140-5p expression in A549, H1299, A549/DDP, H1299/DDP and 16 HBE cells. ${ }^{\star}$ p $<0.05$ or ${ }^{\#}$ p $<0.05$ vs. corresponding control. 
Conversely, miR-140-5p expression was highly decreased by SNHG1 overexpression, while it was increased by SNHG1 knockdown (Figure 3F). All these data implied that SNHG1 might bind to miR-140-5p and repress miR-140-5p expression in DDP-resistant NSCLC cell lines.

Additionally, we measured the expression of miR-140-5p in NSCLC cell lines and 16HBE cells. Of interest, we found that miR-140-5p levels were evidently decreased in DDP-resistant NSCLC cell lines compared with DDP-sensitive NSCLC cell lines (Figure 3G).

si-SNHG1-mediated regulatory effect was antagonized by miR-140-5p in DDP-resistant NSCLC cell lines. It has been reported that lncRNA can act as a molecular sponge of miRNAs, and thus play an important role in many human diseases [17]. Therefore, we asked whether SNHG1 exerted its regulatory effect through miR-140-5p. As shown in Figure 4A, si-SNHG1-mediated inhibition effect on miR-140-5p expression was highly reversed by co-transfection with anti-miR140-5p. Consistently, si-SNHG1-mediated repression effect on IC50 of DDP was evidently abated when co-transfected with anti-miR-140-5p (Figure 4B). Subsequent functional experiments revealed that si-SNHG1-mediated suppression function on cell proliferation, migration and invasion was also drastically antagonized following miR-140-5p expression restoration (Figures $4 \mathrm{C}-4 \mathrm{G}$ ). These data suggested that SNHG1 might exert its regulatory function by sponging miR-140-5p in DDP-resistant NSCLC cell lines.

Wnt/ $\beta$-catenin signaling was involved in SNHG1/ miR-140-5p-mediated regulation in DDP-resistance of NSCLC cell lines. Aberrant activation of $\mathrm{Wnt} / \beta$-catenin signaling was an independent mark correlated with poor prognosis in NSCLC [20]. Additionally, its targets including cyclin D1 and c-Myc were demonstrated to be involved in NSCLC progression [21]. Hence, we identified whether Wnt/ $\beta$-catenin signaling was involved in SNHG1/miR-140$5 \mathrm{p}$-mediated regulation network. Western blot analysis revealed that SNHG1 knockdown blockaded Wnt/ $\beta$-catenin signaling in A549/DDP and H1299/DDP cells, presented as a decrease of Wnt1, cyclinD1, c-Myc and $\beta$-catenin expression levels (Figures 5A and 5B). Whereas, SNHG1-mediated
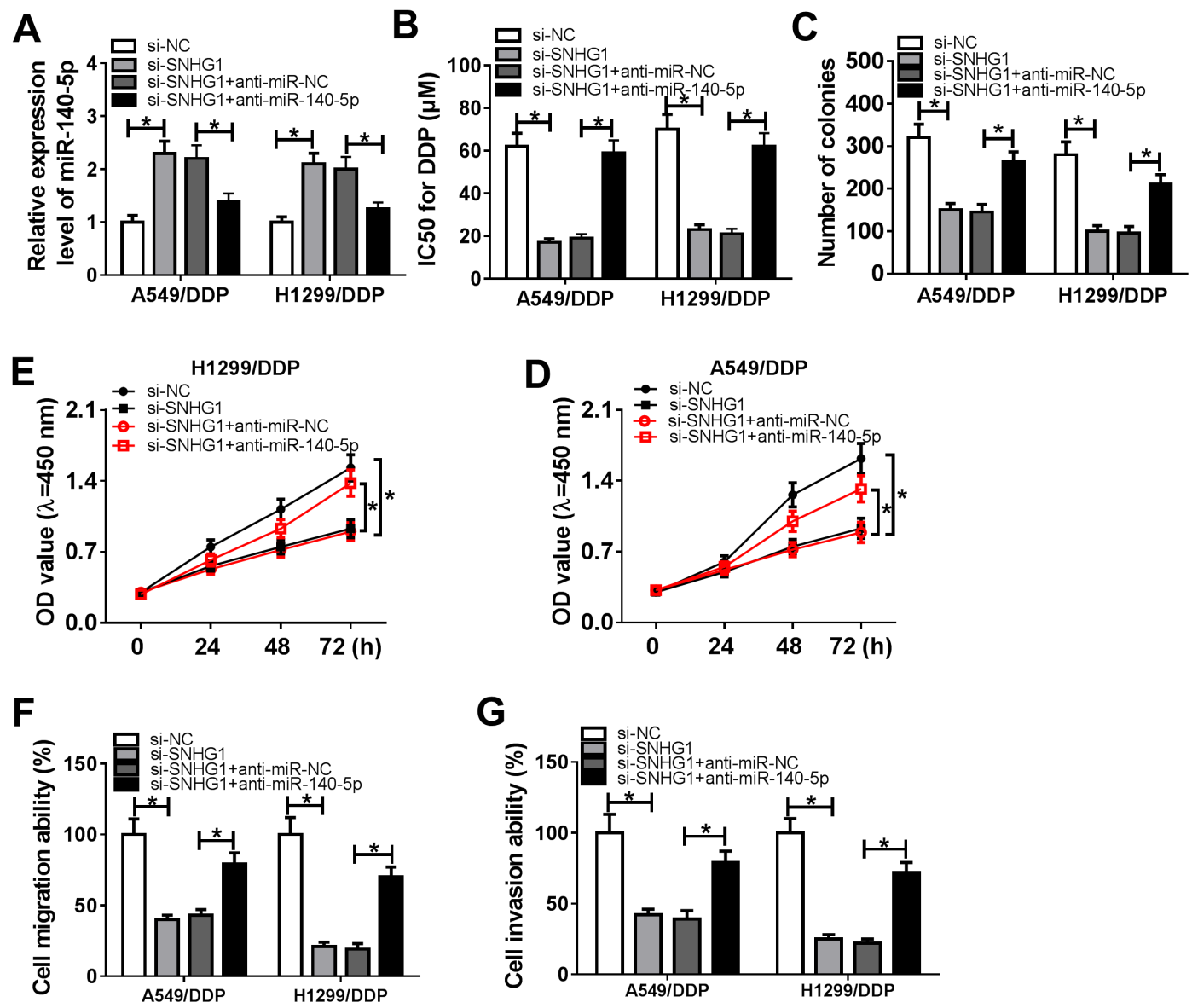

Figure 4. SNHG1 exerted its regulatory function by sponging miR-140-5p. si-NC, si-SNHG1, si-SNHG1+anti-miR-NC, or si-SNHG1+anti-miR-140-5p was transfected into A549/DDP and H1299/DDP cells, followed by the determination of miR-140-5p expression by qRT-PCR assay (A), IC50 of DDP by CCK-8 assay (B), cell colony formation by colony formation assay $(\mathrm{C})$, cell proliferation ability by CCK-8 assay (D) and (E), cell migration (F) and invasion $(G)$ capacities by Transwell assay. ${ }^{*} \mathrm{p}<0.05$ vs. si-NC or si-SNHG1+anti-miR-NC. 


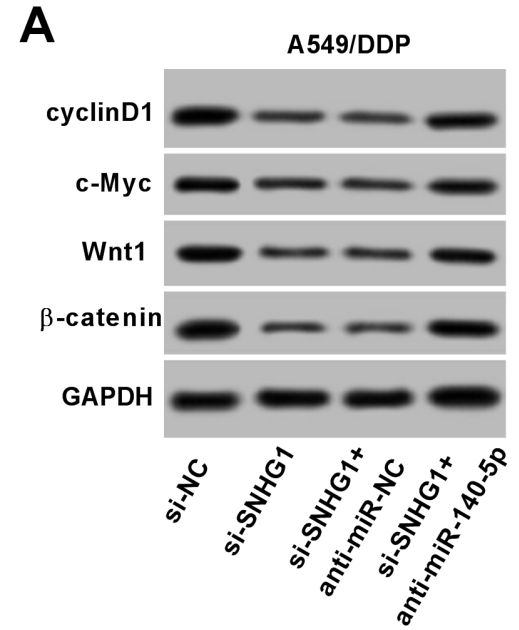

B

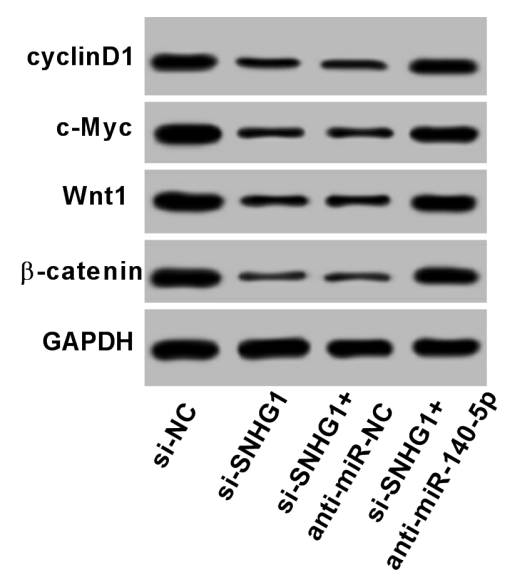

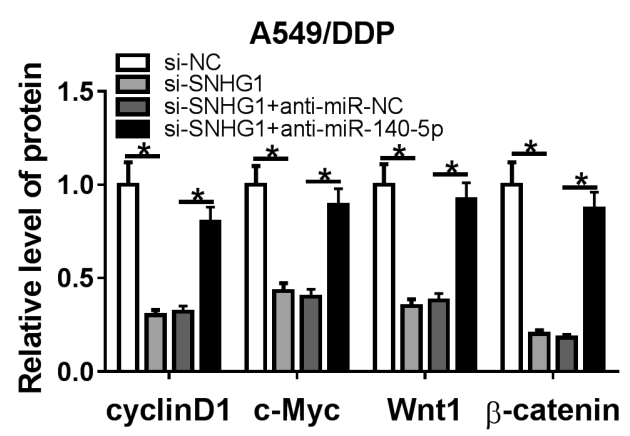

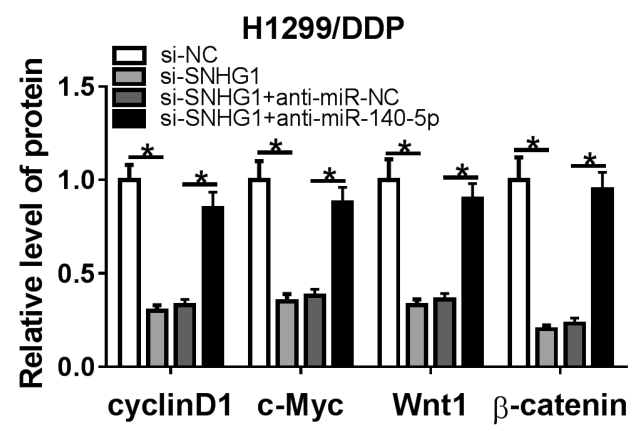

Figure 5. Wnt/ $\beta$-catenin signaling was involved in SNHG1/miR-140-5p-mediated regulation. Western blot analysis was performed to assess the expression levels of Wnt1, cyclinD1, c-Myc and $\beta$-catenin in A549/DDP (A) and H1299/DDP cells (B) transfected with si-NC, si-SNHG1, si-SNHG1+antimiR-NC, or si-SNHG1+anti-miR-140-5p. ${ }^{*} \mathrm{p}<0.05$ vs. si-NC or si-SNHG1+anti-miR-NC.

inhibition effect on the activity of Wnt/ $\beta$-catenin signaling was abolished by anti-miR-140-5p transfection (Figures $5 \mathrm{~A}$ and $5 \mathrm{~B})$. All these results hinted that $\mathrm{Wnt} / \beta$-catenin signaling might be involved in SNHG1/miR-140-5p-mediated regulation in DDP-resistance of NSCLC cell lines.

SNHG1 knockdown repressed tumor growth in vivo. In order to further manifest the function of SNHG1 on DDP-resistance of NSCLC, A549/DDP cells transfected with sh-SNHG1 or sh-NC were subcutaneously implanted into nude mice. All mice underwent an administration of DDP every 5 days and were euthanized after 5 weeks injection. These data demonstrated that SNHG1 knockdown significantly repressed tumor growth, indicated by a decrease of tumor volume (Figure 6A) and tumor weight (Figure 6B). Moreover, miR-140-5p expression was higher in sh-SNHG1 group than that in sh-NC group (Figure 6C). In parallel, SNHG1 depletion markedly blockaded Wnt/ $\beta$-catenin signaling pathway in xenograft tissues (Figures 3D-3E).
Taken together, these data suggested that SNHG1 might promote DDP-resistance of NSCLC by miR-140-5p/Wnt/ $\beta$ catenin pathway in vivo.

\section{Discussion}

The emergence or presence of chemoresistance in tumor cells is a major burden in cancer therapy [22]. Recently, lncRNAs have been widely acknowledged to be implicated in a series of malignant pathological processes, such as tumorigenesis, invasion, metastasis and chemoresistance [23]. Accumulating evidences have suggested that some lncRNAs play critical roles on DDP-resistance of NSCLC. For example, IncRNA CCAT1 accelerated DDP-resistance of NSCLC cells by regulating miR-130a-3p/SOX4 axis [24]. LncRNA AK126698 was demonstrated to enhance DDP-resistance at least partly through targeting $\mathrm{Wnt} / \beta$-catenin signaling pathway [25]. Conversely, upregulation of MEG3 sensitized 
A

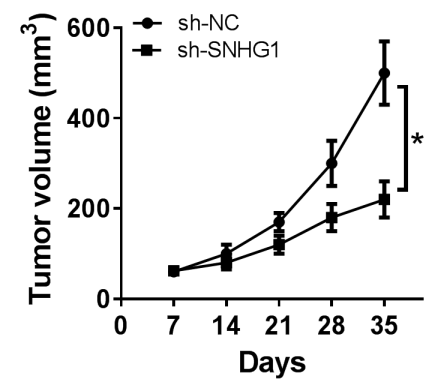

D

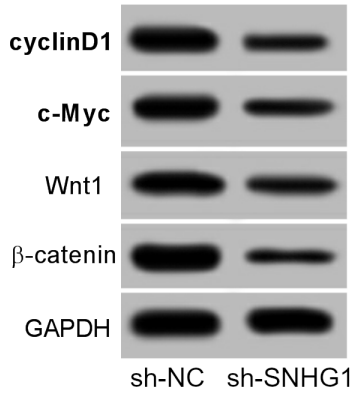

B

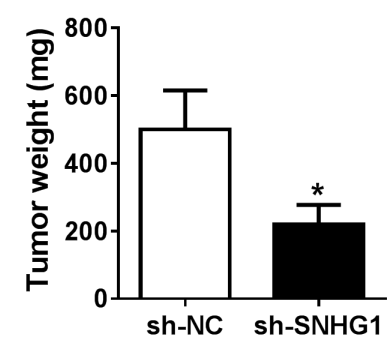

E
C

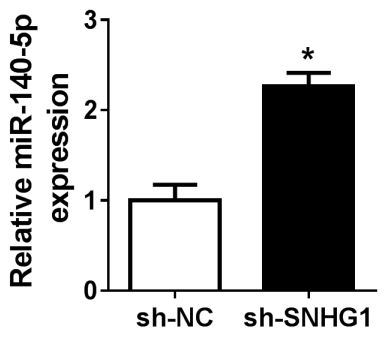

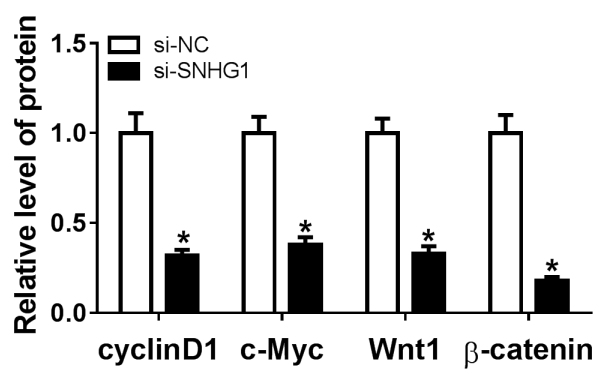

Figure 6. SNHG1 knockdown repressed tumor growth in vivo. Approximately $5.0 \times 10^{6}$ A549/DDP cells stably transfected with lentivirus-mediated shSNHG1 or sh-NC were subcutaneously injected into nude mice ( $n=10)$. At 3 days after implantation, an intravenous administration of DDP solution (3 $\mathrm{mg} / \mathrm{kg}$ ) in each mouse was performed every 5 days. 5 weeks later, xenograft mice were euthanized. A) Tumor volume was measured with a caliper every week after injection 7 days. B) Tumor average weight was measured. C) qRT-PCR assay of miR-140-3p in excised tumor tissues. D and E) Western blot analysis of Wnt1, cyclinD1, c-Myc and $\beta$-catenin expression levels in excised tumor tissues. ${ }^{*} \mathrm{p}<0.05 \mathrm{vs}$. sh-NC.

DDP-resistance in NSCLC cells to cisplatin via regulating the target gene of Wnt/ $\beta$-catenin pathway [26]. GAS5 knockdown weakened the DDP-sensitivity of NSCLC by sponging miR-21 and regulating PTEN pathway [27]. In this study, we found a significant upregulation of SNHG1 expression in DDP-resistance of NSCLC tissues and cell lines. Moreover, SNHG1 knockdown suppressed the DDP-resistance, proliferation, migration and invasion of NSCLC cell lines in vitro, and repressed tumor growth in vivo. All these results implied that SNHG1 might contribute to DDP-resistance of NSCLC.

Recently, the ceRNAs hypothesis proposes that lncRNAs might act as a molecular sponge of miRNAs, and thus implicate in cellular physiopathology processes by directly regulating miRNAs [17]. Hence, bioinformatics prediction softwares were used to predict the directly interactional miRNAs of SNHG1. Among these candidates, miR-140-5p was chosen for further study because it was reported to participate in the development, progression and chemoresistance of multiple human cancers. For instance, miR-140-5p was demonstrated to repress the proliferation of NSCLC cells through MMD/Erk signaling pathway [28]. MiR-140-5p inhibited tumor growth and metastasis of hepatocellular carcinoma by targeting fibroblast growth factor 9 and transforming growth factor $\beta$ receptor 1 [29]. MiR-140-5p overexpression attenuated the proliferation and elevated apoptosis in ovarian cancer by targeting PDGFRA [30]. Besides,
miR-140-5p was described to act as a tumor suppressor in tongue cancer [31], colorectal cancer [32] and squamous cell carcinoma [33]. Moreover, it was reported that miR-140-5p enhanced the chemosensitivity of ovarian cancer cells to DDP [30]. Conversely, miR-140-5p ameliorated DDP/ doxorubicin-induced cell death through targeting IP3k2 in osteosarcoma cells [34]. Here, we demonstrated that SNHG1 repressed miR-140-5p expression by directly binding to miR-140-5p, and si-SNHG1-mediated regulatory effect was antagonized by miR-140-5p. All these results implied that SNHG1 might promote DDP-resistance of NSCLC at least partly by sponging miR-140-5p.

The Wnt/ $\beta$-catenin pathway has an oncogenic role in the development and progression of multiple human cancers such as colorectal cancer [35], prostate carcinoma [36], glioblastoma [37] and chronic myeloid leukemia [38]. Moreover, abnormalities of $\mathrm{Wnt} / \beta$-catenin pathway were demonstrated to be involved in NSCLC progression, for example, Xu et al. [20] and Huang et al. [39] found that Wnt1 or $\beta$-catenin expression levels were closely correlated with poor prognosis of NSCLC patients. In the present study, Wnt/ $\beta$-catenin signaling was found to implicate in SNHG1/ miR-140-5p-mediated regulatory in the DDP-resistance of NSCLC in vitro and in vivo. Consistent with our findings, Wickstrom et al. [40] confirmed that inhibition of Wnt/ $\beta$ catenin signaling downregulated the expression of DNA 
repair enzyme O6-methylguanine-DNA methyltransferase, a regulator of chemoresistance development, and thus restored chemosensitivity of DNA-alkylating drugs in variety human cancers. Moreover, Cui et al. [41] showed that SNHG1 promoted the progression of NSCLC by sponging miR-101-3p and regulating Wnt/ $\beta$-catenin signaling pathway. Additionally, similar with the present finding of lncRNA/ miRNA/signaling pathway regulatory network in DDP-resistance development, CRNDE/miR-181a-5p/Wnt/ $\beta$-catenin signaling [42] and HOTAIR/miR-218/NF- $\kappa \mathrm{B}$ signaling [43] were also demonstrated to participate in the development of chemoresistance in human cancers.

In conclusion, our data demonstrated that SNHG1 contributed to DDP-resistance of NSCLC at least in part through regulating $\mathrm{miR}-140-5 \mathrm{p} / \mathrm{Wnt} / \beta$-catenin signaling, providing a novel lncRNA/miRNA/signaling pathway regulatory network involved in DDP-resistance of NSCLC.

\section{References}

[1] FERLAY J, SOERJOMATARAM I, DIKSHIT R, ESER S, MATHERS $\mathrm{C}$ et al. Cancer incidence and mortality worldwide: sources, methods and major patterns in GLOBOCAN 2012. Int J Cancer 2015; 136: E359-386. https://doi. org/10.1002/ijc.29210

[2] GRIDELLI C, ROSSI A, CARBONE DP, GUARIZE J, KARACHALIOU $\mathrm{N}$ et al. Non-small-cell lung cancer. Nat Rev Dis Primers 2015; 1: 15009. https://doi.org/10.1038/ nrdp.2015.9

[3] OLAUSSEN KA, DUNANT A, FOURET P, BRAMBILLA E, ANDRE F et al. DNA repair by ERCC1 in non-small-cell lung cancer and cisplatin-based adjuvant chemotherapy. $\mathrm{N}$ Engl J Med 2006; 355: 983-991. https://doi.org/10.1056/ NEJMoa060570

[4] SARIN N, ENGEL F, KALAYDA GV, MANNEWITZ M, CINATL J JR. et al. Cisplatin resistance in non-small cell lung cancer cells is associated with an abrogation of cisplatin-induced G2/M cell cycle arrest. PLoS One 2017; 12: e0181081. https://doi.org/10.1371/journal.pone.0181081

[5] VANCE KW, PONTING CP. Transcriptional regulatory functions of nuclear long noncoding RNAs. Trends Genet 2014; 30: 348-355. https://doi.org/10.1016/j.tig.2014.06.001

[6] SCHMITT AM, CHANG HY. Long Noncoding RNAs in Cancer Pathways. Cancer Cell 2016; 29: 452-463. https://doi. org/10.1016/j.ccell.2016.03.010

[7] SHI H, PU J, ZHOU XL, NING YY, BAI C. Silencing long non-coding RNA ROR improves sensitivity of non-small-cell lung cancer to cisplatin resistance by inhibiting PI3K/Akt/mTOR signaling pathway. Tumour Biol 2017; 39: 1010428317697568. https://doi. org/10.1177/1010428317697568

[8] WANG P, CHEN D, MA H, LI Y. LncRNA MEG3 enhances cisplatin sensitivity in non-small cell lung cancer by regulating miR-21-5p/SOX7 axis. Onco Targets Ther 2017; 10: 5137-5149. https://doi.org/10.2147/ott.s146423
[9] SUN Y, WEI G, LUO H, WU W, SKOGERBO G et al. The long noncoding RNA SNHG1 promotes tumor growth through regulating transcription of both local and distal genes. Oncogene 2017; 36: 6774-6783. https://doi. org/10.1038/onc.2017.286

[10] WANG Q, LI Q, ZHOU P, DENG D, XUE L et al. Upregulation of the long non-coding RNA SNHG1 predicts poor prognosis, promotes cell proliferation and invasion, and reduces apoptosis in glioma. Biomed Pharmacother 2017; 91: 906-911. https://doi.org/10.1016/j.biopha.2017.05.014

[11] ZHANG H, ZHOU D, YING M, CHEN M, CHEN P et al. Expression of Long Non-Coding RNA (lncRNA) Small Nucleolar RNA Host Gene 1 (SNHG1) Exacerbates Hepatocellular Carcinoma Through Suppressing miR-195. Med Sci Monit 2016; 22: 4820-4829.

[12] JIANG Z, JIANG C, FANG J. Up-regulated lnc-SNHG1 contributes to osteosarcoma progression through sequestration of miR-577 and activation of WNT2B/Wnt/beta-catenin pathway. Biochem Biophys Res Commun 2018; 495: 238245. https://doi.org/10.1016/j.bbrc.2017.11.012

[13] YOU J, FANG N, GU J, ZHANG Y, LI X et al. Noncoding RNA small nucleolar RNA host gene 1 promote cell proliferation in nonsmall cell lung cancer. Indian J Cancer 2014; 51 Suppl 3: e99-e102. https://doi.org/10.4103/0019509x.154092

[14] LU Q, SHAN S, LI Y, ZHU D, JIN W et al. Long noncoding RNA SNHG1 promotes non-small cell lung cancer progression by up-regulating MTDH via sponging miR-1455p. FASEB J 2018; 32: 3957-3967.https://doi.org/10.1096/ f.201701237RR

[15] VIDIGAL JA, VENTURA A. The biological functions of miRNAs: lessons from in vivo studies. Trends Cell Biol 2015; 25: 137-147. https://doi.org/10.1016/j.tcb.2014.11.004

[16] BITARTE N, BANDRES E, BONI V, ZARATE R, RODRIGUEZ J et al. MicroRNA-451 is involved in the self-renewal, tumorigenicity, and chemoresistance of colorectal cancer stem cells. Stem Cells 2011; 29: 1661-1671. https://doi. org/10.1002/stem.741

[17] KARTHA RV, SUBRAMANIAN S. Competing endogenous RNAs (ceRNAs): new entrants to the intricacies of gene regulation. Front Genet 2014; 5: 8. https://doi.org/10.3389/ fgene.2014.00008

[18] YUE B, CAI D, LIU C, FANG C, YAN D. Linc00152 Functions as a Competing Endogenous RNA to Confer Oxaliplatin Resistance and Holds Prognostic Values in Colon Cancer. Mol Ther 2016; 24: 2064-2077. https://doi.org/10.1038/ mt.2016.180

[19] JIANG Z, YIN J, FU W, MO Y, PAN Y et al. MiRNA 17 family regulates cisplatin-resistant and metastasis by targeting TGFbetaR2 in NSCLC. PLoS One 2014; 9: e94639. https:// doi.org/10.1371/journal.pone.0094639

[20] XU X, SUN PL, LI JZ, JHEON S, LEE CT et al. Aberrant Wnt1/beta-catenin expression is an independent poor prognostic marker of non-small cell lung cancer after surgery. J Thorac Oncol 2011; 6: 716-724. https://doi.org/10.1097/ JTO.0b013e31820c5189 
[21] WANG Y, BAI W, WANG M, YU T, ZHANG W. Long NonCoding RNA Brain Cytoplasmic RNA 1 Acts as an Oncogene and Regulates Cell Proliferation and Metastasis in NonSmall Cell Lung Cancer. J Nanosci Nanotechnol 2019; 19: 1978-1985. https://doi.org/10.1166/jnn.2019.16402

[22] HOUSMAN G, BYLER S, HEERBOTH S, LAPINSKA K, LONGACRE $\mathrm{M}$ et al. Drug Resistance in Cancer: An Overview. Cancers 2014; 6: 1769-1792. https://doi.org/10.3390/ cancers6031769

[23] MAJIDINIA M, YOUSEFI B. Long non-coding RNAs in cancer drug resistance development. DNA Repair (Amst) 2016; 45: 25-33. https://doi.org/10.1016/j.dnarep.2016.06.003

[24] HU B, ZHANG H, WANG Z, ZHANG F, WEI H et al. LncRNA CCAT1/miR-130a-3p axis increases cisplatin resistance in non-small-cell lung cancer cell line by targeting SOX4. Cancer Biol Ther 2017; 18: 974-983. https://doi.org/1 0.1080/15384047.2017.1385679

[25] YANG Y, LI H, HOU S, HU B, LIU J et al. The noncoding RNA expression profile and the effect of lncRNA AK126698 on cisplatin resistance in non-small-cell lung cancer cell. PLoS One 2013; 8: e65309. https://doi.org/10.1371/journal. pone.0065309

[26] XIA Y, HE Z, LIU B, WANG P, CHEN Y. Downregulation of Meg3 enhances cisplatin resistance of lung cancer cells through activation of the WNT/beta-catenin signaling pathway. Mol Med Rep 2015; 12: 4530-4537. https://doi. org/10.3892/mmr.2015.3897

[27] CAO L, CHEN J, OU B, LIU C, ZOU Y et al. GAS5 knockdown reduces the chemo-sensitivity of non-small cell lung cancer (NSCLC) cell to cisplatin (DDP) through regulating miR-21/PTEN axis. Biomed Pharmacother 2017; 93: 570579. https://doi.org/10.1016/j.biopha.2017.06.089

[28] LI W, HE F. Monocyte to macrophage differentiation-associated (MMD) targeted by miR-140-5p regulates tumor growth in non-small cell lung cancer. Biochem Biophys Res Commun 2014; 450: 844-850. https://doi.org/10.1016/j. bbrc.2014.06.075

[29] YANG H, FANG F, CHANG R, YANG L. MicroRNA-140-5p suppresses tumor growth and metastasis by targeting transforming growth factor beta receptor 1 and fibroblast growth factor 9 in hepatocellular carcinoma. Hepatology 2013; 58: 205-217. https://doi.org/10.1002/hep.26315

[30] LAN H, CHEN W, HE G, YANG S. miR-140-5p inhibits ovarian cancer growth partially by repression of PDGFRA. Biomed Pharmacother 2015; 75: 117-122. https://doi. org/10.1016/j.biopha.2015.07.035

[31] KAI Y, PENG W, LING W, JIEBING H, ZHUAN B. Reciprocal effects between microRNA-140-5p and ADAM10 suppress migration and invasion of human tongue cancer cells. Biochem Biophys Res Commun 2014; 448: 308-314. https:// doi.org/10.1016/j.bbrc.2014.02.032

[32] ZHANG W, ZOU C, PAN L, XU Y, QI W et al. MicroRNA140-5p inhibits the progression of colorectal cancer by targeting VEGFA. Cell Physiol Biochem 2015; 37: 1123-1133. https://doi.org/10.1159/000430237
[33] JING P, SA N, LIU X, LIU X, XU W. MicroR-140-5p suppresses tumor cell migration and invasion by targeting ADAM10-mediated Notch1 signaling pathway in hypopharyngeal squamous cell carcinoma. Exp Mol Pathol 2016; 100: 132-138. https://doi.org/10.1016/j.yexmp.2015.12.008

[34] WEI R, CAO G, DENG Z, SU J, CAI L. miR-140-5p attenuates chemotherapeutic drug-induced cell death by regulating autophagy through inositol 1,4,5-trisphosphate kinase 2 (IP3k2) in human osteosarcoma cells. Biosci Rep 2016; 36. https://doi.org/10.1042/bsr20160238

[35] SUZUKI H, WATKINS DN, JAIR KW, SCHUEBEL KE, MARKOWITZ SD et al. Epigenetic inactivation of SFRP genes allows constitutive WNT signaling in colorectal cancer. Nat Genet 2004; 36: 417-422. https://doi.org/10.1038/ ng1330

[36] CHEN G, SHUKEIR N, POTTI A, SIRCAR K, APRIKIAN A et al. Up-regulation of Wnt-1 and beta-catenin production in patients with advanced metastatic prostate carcinoma: potential pathogenetic and prognostic implications. Cancer 2004; 101: 1345-1356. https://doi.org/10.1002/cncr.20518

[37] KAUR N, CHETTIAR S, RATHOD S, RATH P, MUZUMDAR D et al. Wnt3a mediated activation of Wnt/beta-catenin signaling promotes tumor progression in glioblastoma. Mol Cell Neurosci 2013; 54: 44-57. https://doi.org/10.1016/j. mcn.2013.01.001

[38] CORREA S, BINATO R, DU ROCHER B, CASTELOBRANCO MT, PIZZATTI L et al. Wnt/beta-catenin pathway regulates $\mathrm{ABCB} 1$ transcription in chronic myeloid leukemia. BMC Cancer 2012; 12: 303. https://doi.org/10.1186/14712407-12-303

[39] HUANG CL, LIU D, ISHIKAWA S, NAKASHIMA T, NAKASHIMA $\mathrm{N}$ et al. Wnt1 overexpression promotes tumour progression in non-small cell lung cancer. Eur J Cancer 2008; 44: 2680-2688. https://doi.org/10.1016/j.ejca.2008.08.004

[40] WICKSTROM M, DYBERG C, MILOSEVIC J, EINVIK C, CALERO $\mathrm{R}$ et al. Wnt/ $\beta$-catenin pathway regulates MGMT gene expression in cancer and inhibition of Wnt signalling prevents chemoresistance. Nature Communications 2015; 6: 8904. https://doi.org/10.1038/ncomms9904

[41] CUI Y, ZHANG F, ZHU C, GENG L, TIAN T et al. Upregulated lncRNA SNHG1 contributes to progression of nonsmall cell lung cancer through inhibition of miR-101-3p and activation of Wnt/beta-catenin signaling pathway. Oncotarget 2017, 8: 17785-17794. http://doi.org/10.18632/oncotarget.14854

[42] HAN P, LI JW, ZHANG BM, LV JC, LI YM et al. The lncRNA CRNDE promotes colorectal cancer cell proliferation and chemoresistance via miR-181a-5p-mediated regulation of Wnt/beta-catenin signaling. Mol Cancer 2017; 16: 9. https:// doi.org/10.1186/s12943-017-0583-1

[43] LI P, ZHANG X, WANG L, DU L, YANG Y et al. lncRNA HOTAIR Contributes to 5FU Resistance through Suppressing miR-218 and Activating NF-kappaB/TS Signaling in Colorectal Cancer. Mol Ther Nucleic Acids 2017; 8: 356-369. https://doi.org/10.1016/j.omtn.2017.07.007 